\title{
Variabilidad temporal de pequeña escala en el fitoplancton de bahía independencia, Pisco, Perú
}

\author{
Small scale temporal variability in the phytoplankton of Independencia Bay, \\ Pisco, Perú
}

Noemí Ochoa y Juan Tarazona*

Presentado: $17 / 06 / 2003$

Aceptado: 07/07/2003

\section{Resumen}

Se estudió la variación temporal a pequeña escala de la comunidad fitoplanctónica costera en una estación fija en Bahía Independencia. Las muestras fueron colectadas del 28 de marzo al 25 de abril de 1988, dos veces al día, a las 08:00 y 15:00 horas. La temperatura superficial del mar fluctuó entre 15,4 a $17,2^{\circ} \mathrm{C}$. Las diatomeas fueron el principal componente de la comunidad fitoplanctonica y estuvieron relacionadas con el total de fitoplancton. Otros grupos tales como los dinoflagelados, cocolitofóridos, silicoflagelados y microflagelados fueron de menor importancia. Durante los primeros nueve días la especie dominante fue Skeletonema costatum, la que fue sustituida posteriormente por Thalassionema nitzschioides hasta el final del estudio. Se encontró escasa variabilidad en composición de especies, pero muy alta en densidad en períodos de sólo algunas horas. Los cambios en la estructura fitoplanctónica estuvieron relacionados con pequeños cambios de la temperatura.

Palabras clave: Fitoplancton, diatomeas, variación temporal, Bahía Independencia, Perú.

\section{Abstract}

Temporal variations at small scale of the coastal marine phytoplankton assemblages were studied. Water samples were collected at a fixed station in Bahia Independencia (Pisco-Peru). The sampling took place in the morning $(08: 00 \mathrm{~h}$.) and afternoon $(15: 00 \mathrm{~h})$ over a period of 29 days (March 28 to April $25,1988)$. Surface temperatures also were taken, fluctuating from $15,4{ }^{\circ} \mathrm{C}$ to $17,2{ }^{\circ} \mathrm{C}$.

Diatoms were the principal component of the phytoplankton community and were more related with the total of phytoplankton. Other groups as Dinoflagellates, Coccolitophorids, Silicoflagellates and small flagellates were present but were less important. Skeletonema costatum was the dominant specie during the first nine days of sampling, after that it was substituted by Thalassionema nitzschioides, which remained as dominant until the end of the study. Small variation in species composition but large fluctuations in density of phytoplankton were recorded over a period of few hours. Small increments in temperature influenced in the phytoplankton assemblages.

Keywords: Phytoplankton, Diatoms, temporal variation, Independencia Bay, Perú.

\section{Introducción}

La alta producción fitoplanctónica registrada en el área de Pisco sustenta la importante actividad extractiva de peces y mariscos para consumo humano. Es por ello que los estudios sobre las características y dinámica de la comunidad fitoplanctónica concita el interés de científico y administradores ya que permite conocer mejor el funcionamiento del ecosistema y de sus recursos.

\footnotetext{
* Facultad de Ciencias Biológicas, Universidad Nacional Mayor de San Marcos. Apartado 1898. Lima 100 Perú. eMail:
}

Noemi Ochoa: aochoal@unmsm.edu.pe.
Estudios sobre variación espacial y estacional del fitoplancton en esta área han sido efectuados por Barreda (1958), Sánchez et al. (1988), Antonietti (1989) y Tarazona et al. (1989).

El principal objetivo de este estudio es analizar la variabilidad temporal del fitoplancton a nivel de su estructura comunitaria y especies dominantes, en períodos cortos de tiempo en una estación fija en Bahía Independencia, Pisco, Perú. 


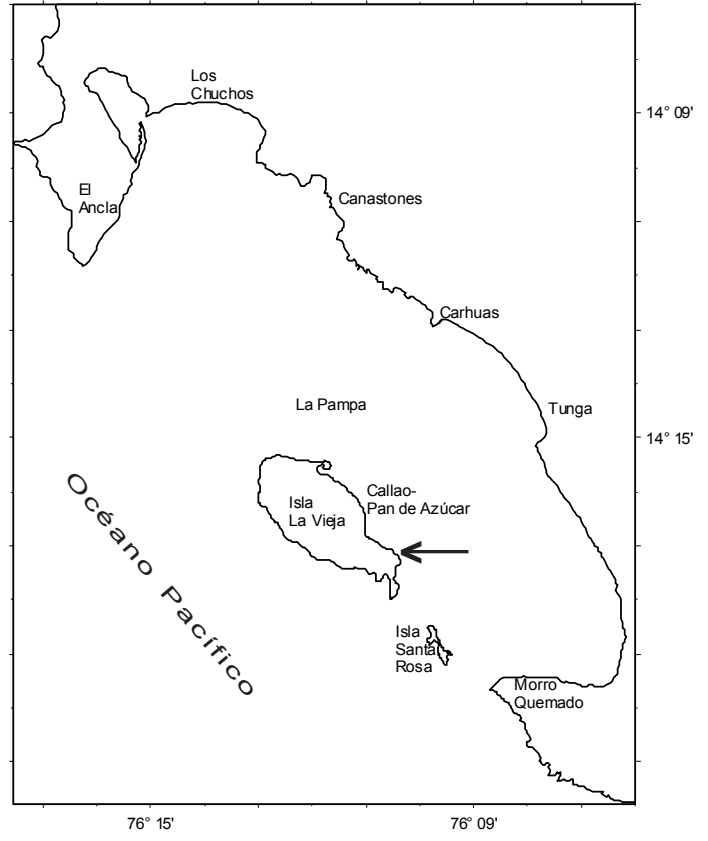

Figura 1. Mapa de Bahía Independencia, la flecha indica el lugar de muestreo.

\section{Material y métodos}

Del 28 de marzo al 25 de abril de 1988, se colectaron 56 muestras de agua superficial utilizando una botella Niskin, en una estación situada a un metro de la orilla en la isla Inde- pendencia, Pisco, $\left(76^{\circ} 08^{\circ} \mathrm{W}-14^{\circ} 15^{\prime} \mathrm{S}\right)$, (Fig. 1). Los muestreos se realizaron dos veces al día, a las 08:00 y 15:00 horas, simultáneamente se registraron las temperaturas superficiales del mar.

El análisis cuantitativo de fitoplancton siguió el método de sedimentación de Utermöhl (1958) y las recomendaciones de UNESCO, (1978), utilizándose cámaras de sedimentación de $50 \mathrm{~mL}$. La densidad del fitoplancton fue expresada en número de células por $50 \mathrm{~mL}$.

Los organismos, en lo posible, fueron identificados hasta el nivel de especie; en el caso de los microflagelados no identificables debido a su pequeño tamaño, fueron reunidos en un grupo, como "fitoflagelados".

El índice de diversidad ( $\left.\mathrm{H}^{\prime}\right)$ se calculó aplicando la fórmula de Shannon y Wiener (1963). Para el análisis de similitud entre muestras se utilizó el índice de Bray Curtis. Previamente los valores de densidad fueron transformados a $\log (n+1)$. Los valores de similitud obtenidos fueron sometidos a un análisis de ordenación no-métrica multidimensional (MDS) y a un análisis de clasificación jerárquica en modo Q (entre estaciones) y su dendrograma fue

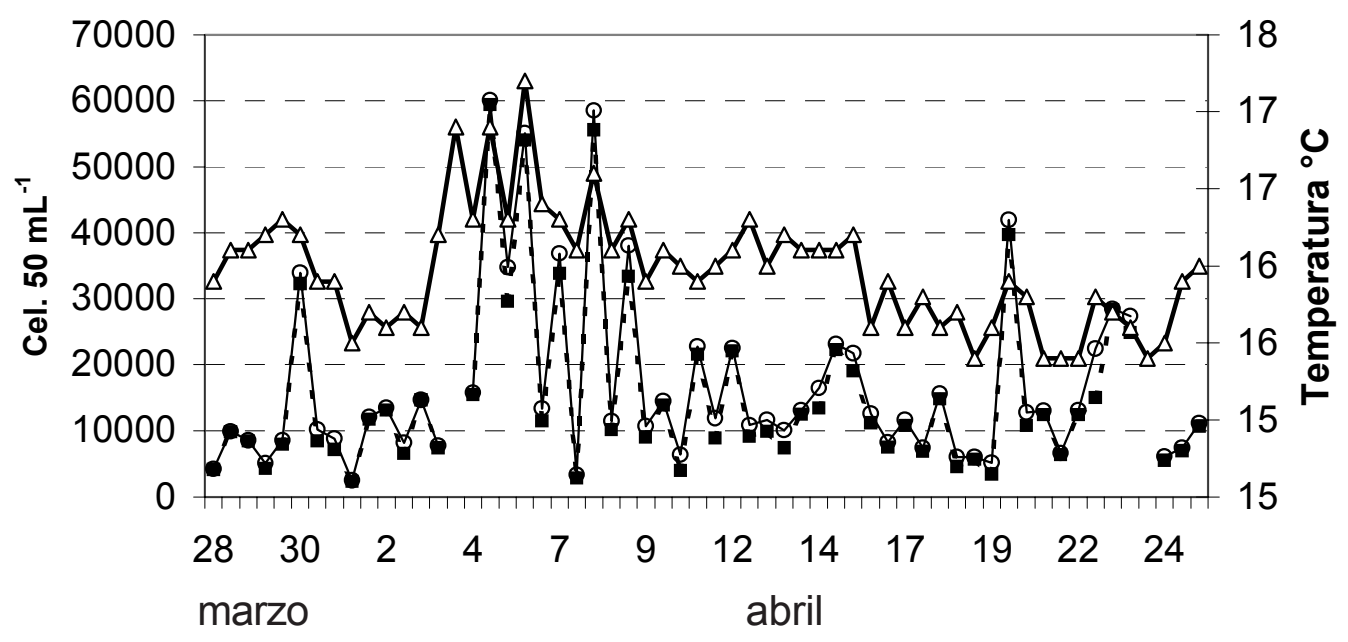

- - Diatomeas -0 -Total de fitoplancton $\triangle-$ Temperatura

Figura 2. Variación temporal de la temperatura superficial $\left({ }^{\circ} \mathrm{C}\right)$, total de fitoplancton y diatomeas (cel.50 mL-1) en Bahía Independencia. Cada día incluye dos puntos, el primero corresponde a las 08:00 horas y el segundo a las 15:00 horas. 


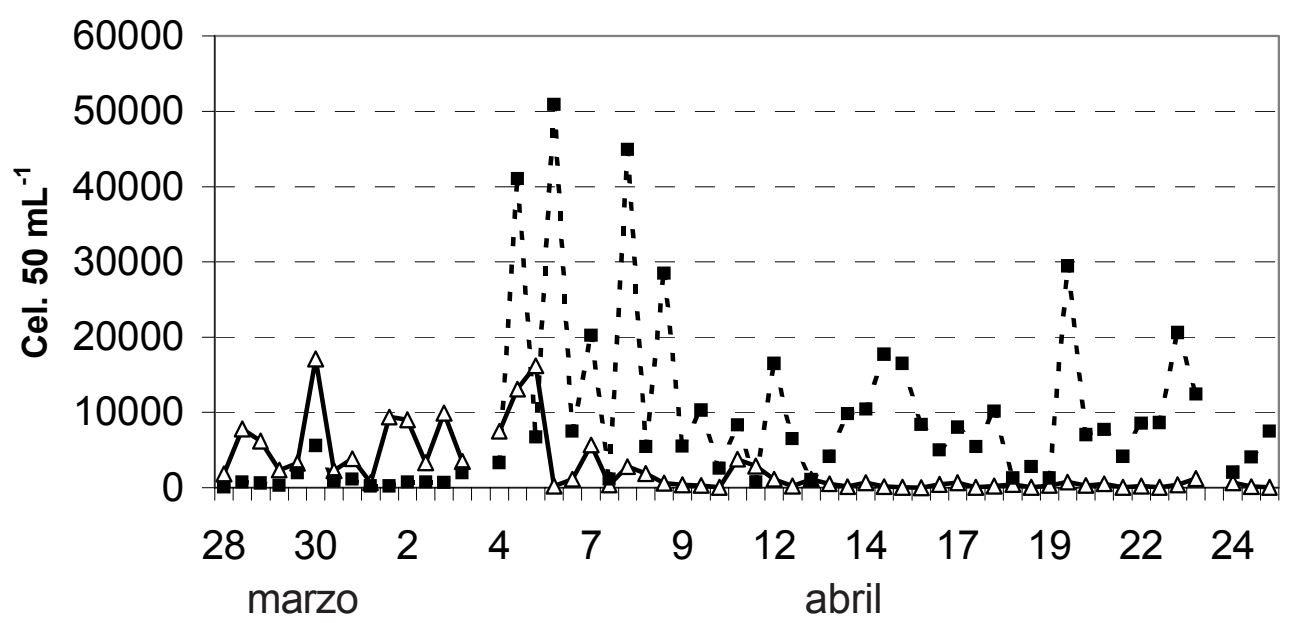

$\triangle-$ Skeletonema costatum - - Thalassionema nitzschioides

Figura 3. Variación temporal de Thalassionema nitzschioides y Skeletonema costatum ( cel. $50 \mathrm{~mL}^{-1}$ ) en Bahía Independencia. Cada dia incluye dos puntos, el primero corresponde a las 08:00 horas y el segundo a las 15:00 horas.

construído utilizando el método UPGMA, Sneath y Sokal (1973).

\section{Resultados}

\section{Temperatura}

Las temperaturas registradas fluctuaron entre $15,4{ }^{\circ} \mathrm{C}$ y $17,2^{\circ} \mathrm{C}$. Las mayores temperaturas se registraron del 3 al 9 de mayo, observándose valores mayores a $16,2^{\circ} \mathrm{C}$ (Fig. 2).

\section{Composición y abundancia del fitoplancton}

Se identificaron 114 especies, de las cuales 68 fueron diatomeas, 38 dinoflagelados, 2 cocolitoforidos, 2 silicoflageladosy 4 microflagelados.

La densidad del fitoplancton varió entre 2540 y 59669 cel.50 $\mathrm{mL}^{-1}$, con un valor pro-

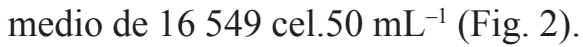

Las diatomeas fueron el grupo dominante y constituyeron el $91,53 \%$ del total de fitoplancton (Fig. 2), con densidades que fluctuaron entre 2294 y 58977 cel.50 mL $\mathrm{mL}^{-1}$. La especie Thalassionema nitzschioides fue la más abundante, representando el 54,65\% del total de fitoplancton, estuvo presente durante todo el período de estudio y se constituyó en la especie dominante a partir del 6 de abril, décimo día del estudio (Fig. 3). La segunda especie en abundancia fue Skeletonema costatum, que constityó el 16\% de la población fitoplanctónica; esta especie fue dominante en los primeros nueve dias y disminuyó drásticamente a partir del décimo dia pero sin llegar a desaparecer, ya que su frecuencia fue del 100\% (Fig. 3).

Otras diatomeas importantes por su frecuencia, presentes en más del 90\% de las muestras, aunque de bajas densidades fueron, Thalassionema bacillaris, Asterionellopsis glacialis, Guinardia delicatula, Detonula pumila, Pleurosigma nicobaricum, Thalassiosira anguste lineata, Thalassiosira mendiolana, Grammatophora marina, Amphiprora sp., Gyrosigma sp. y Navicula sp.

Los fitoflagelados constituyeron sólo el 6\% del total de fitoplancton; sin embargo fueron muy frecuentes y estuvieron presentes en el $90 \%$ de las muestras. Igualmente los dinoflagelados cuantitativamente no fueron importantes pero sí frecuentes, especialmente Ceratium fusus var. fusus, Gymnodinium sanguineum, Ceratium furca, Ceratium tripos, Dinophysis acuminata, Protoperidinium obtusum y Scripsiella trochoidea. 


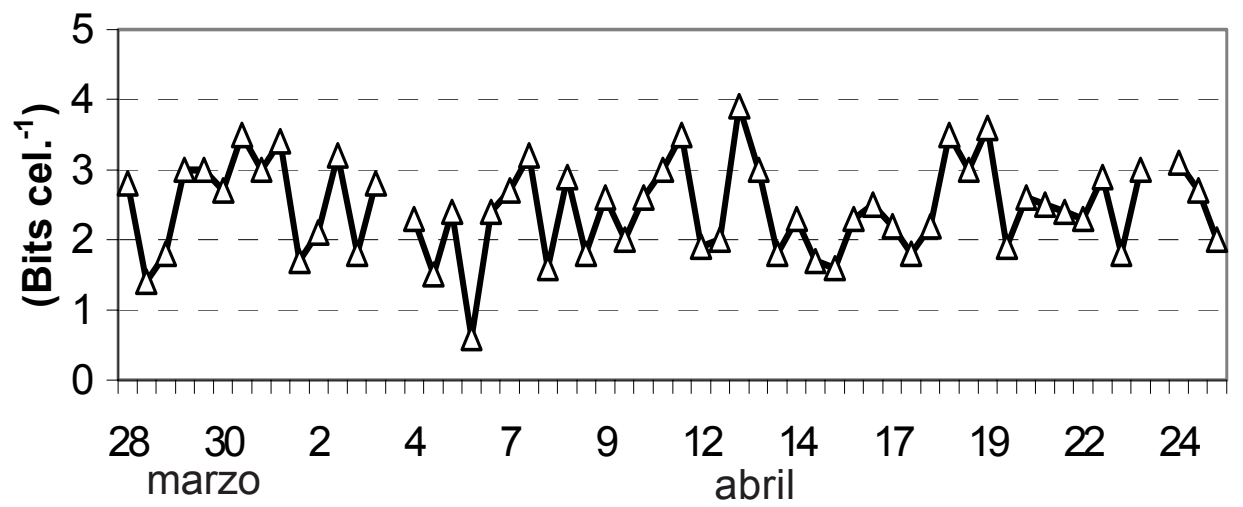

Figura 4. Variación temporal del índice de diversidad H' (Bits cel. ${ }^{-1}$ ) en Bahía Independencia. Cada dia incluye dos puntos, el primero corresponde a las 08:00 horas y el segundo a las 15:00 horas.

La presencia de cocolitoforidos y silicoflagelados no fue significativa; juntos constituyeron sólo el 0,01\% de la población. El índice de diversidad fue bajo, con valores generalmente menores de 3 bits por individuo (Fig. 4).

\section{Variación temporal}

La distribución de la densidad total de fitoplancton a las 08:00 y 15:00 horas (Fig. 2) mostró una variabilidad muy grande pasando de valores máximos a mínimos y viceversa. En general el fitoplancton fue más denso a las 8:00 horas, con un valor promedio de 20685 cel.50 $\mathrm{mL}^{-1}$, mientras que a las 15:00 horas fue de 12816 cel.50 mL $\mathrm{mL}^{-1}$ (Fig. 5); esta misma tendencia se presentó en la especie más abundante, T. nitzschioides (Fig. 6). S. costatum la segunda especie en abundancia, en cambio, fue ligeramente más abundante a las 15:00 horas (Fig. 7). Algunas especies mostraron un patrón de distribución similar al de $T$. nizschioides, tales como T. bacillaris, C. fusus var fusus y G. sanguineum; otras siguieron el patron de $S$. costatum, entre ellas, A. glacialis.

Los promedios diarios de densidad de fitoplancton total (Fig. 8) y de las principales especies mostraron el mismo carácter oscilatorio que la densidad del fitoplancton a las 08:00 y 15:00 horas. Durante los primeros nueve días, $S$. costatum fue la especie dominante y contribuyó con un máximo de 10162 cel. $50 \mathrm{~mL}^{-1}$ al primer pico de fitoplancton; posteriormente fue reemplazada por $T$.

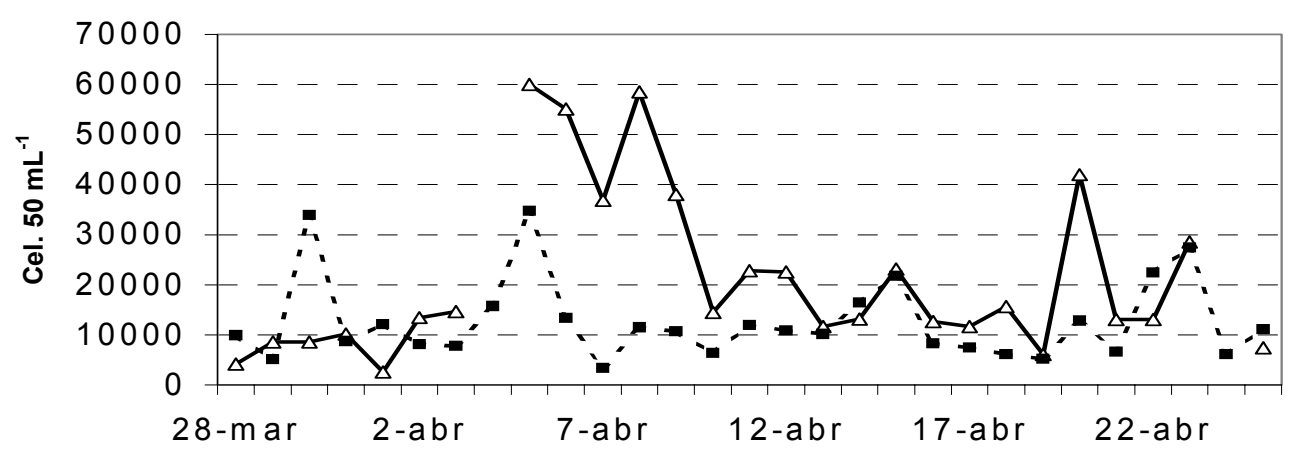

$\checkmark-8: 00$ horas - $-15: 00$ Horas

Figura 5. Variación diaria del total de fitoplancton (cel.50 $\mathrm{mL}^{-1}$ ) en Bahía Independencia a las 8:00 y 15:00 horas. 


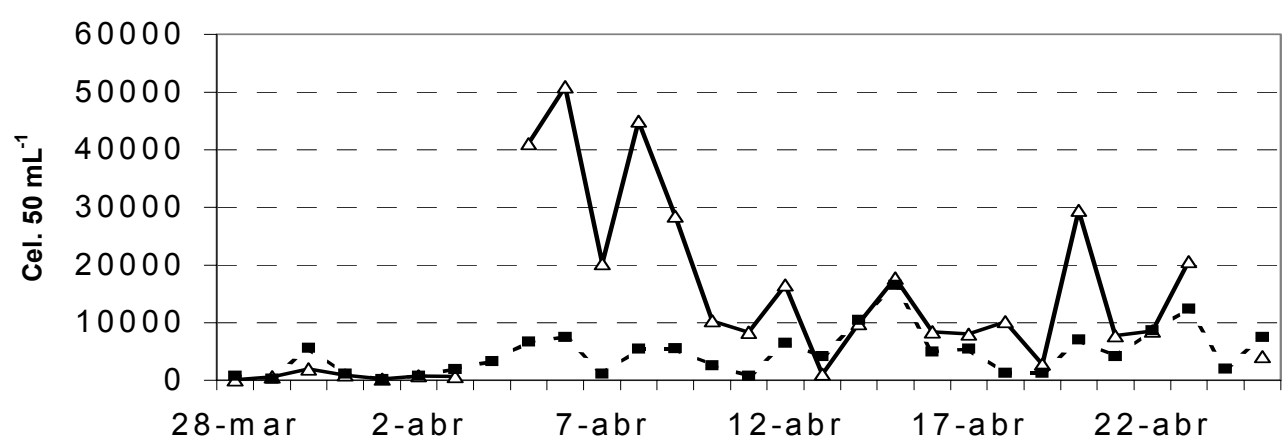

$\triangle-8: 00$ hHoras $--15: 00$ Horas

Figura 6. Variación diaria de Thalassionema nitzschioides (cel.50 $\mathrm{mL}^{-1}$ ) a las $8: 00$ y 15:00 horas.

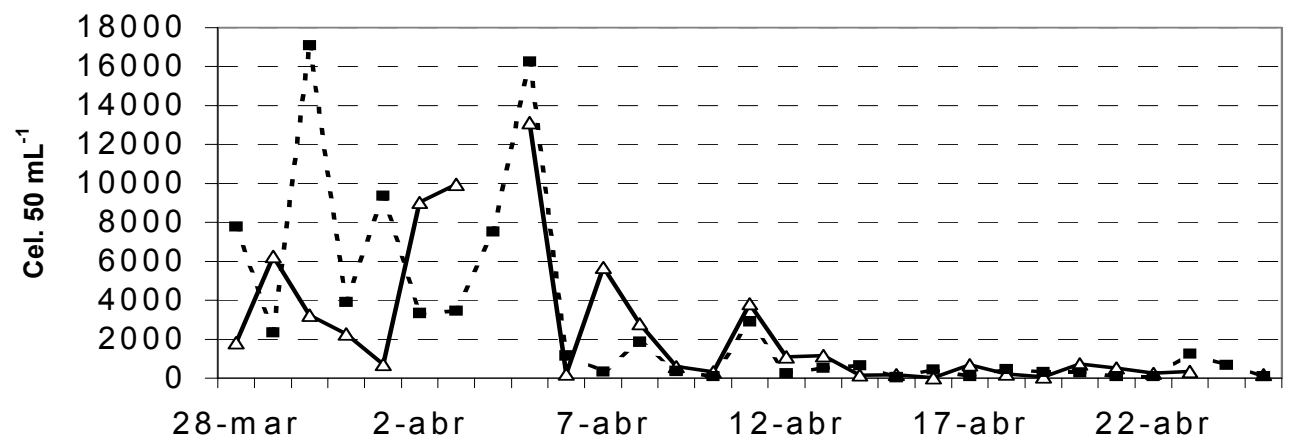

$\triangle-8: 00$ Horas $--15: 00$ Horas

Figura 7. Variación diaria de Skeletonema costatum (cel.50 $\left.\mathrm{mL}^{-1}\right)$ a las 8:00 y 15:00 horas.

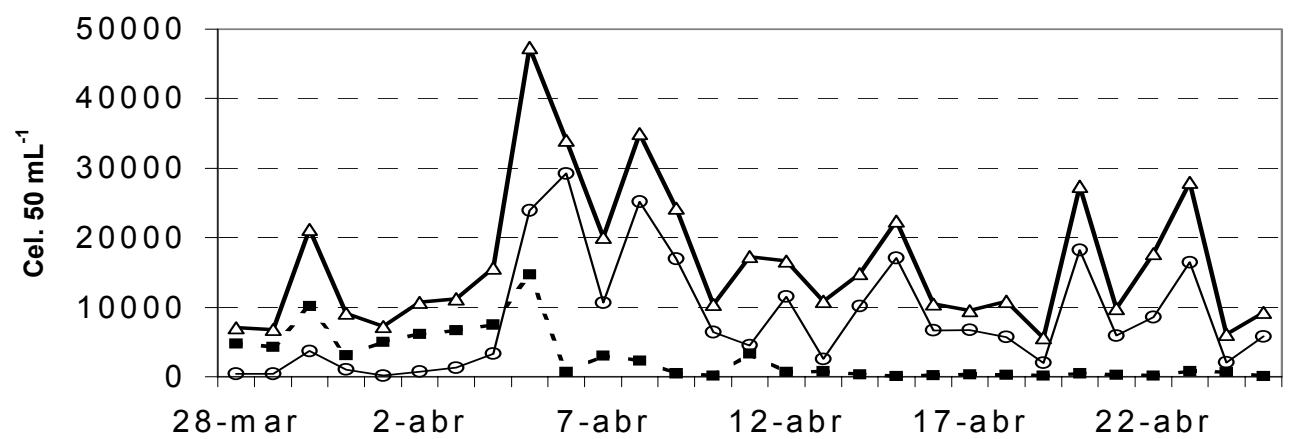

$\triangle \triangle$ Total de fitoplancton - - Skeletonema costatum -0 - Thalassionema nitzschioides

Figura 8. Variación del promedio diario de densidad del total de fitoplancton, Skeletonema costatum y Thalassionema nitzschioides (cel.50 $\mathrm{mL}^{-1}$ ) 
nitzschioides, que alcanzó un máximo de 29 225 cel.50 mL $\mathrm{mL}^{-1}$ (Fig. 3).

Los porcentajes de similitud entre muestras fueron altos(Fig. 9), no obstante la variabilidad del fitoplancton. Se discriminaron dos bloques; uno integrado por las muestras de los primeros 9 días hasta el 5 de abril, caracterizado por la dominancia de $S$. costatum con $57 \%$ de contribución, seguida de $T$. nitzschioides con $11 \%$, A. glacialis con $6 \%$ y fitoflagelados con $4 \%$ y otro bloque constituido por las muestras del 9 al 22 de abril con dominancia de $T$. nitzschioides con $66 \%$ de contribución, seguido de fitoflagelados con $8 \%$, Thalassiosira anguste lineata con 4\%,T. bacillaris 4\% y Thalassiosira mendiolana $2 \%$. Las muestras restantes que corresponden a un período intermedio entre los dos bloques y a las muestras de los últimos dias, forman pequeños grupos separados o se encuentran aisladas de las demás.

El ajuste multidimensional no métrico, NMDS, (Fig. 10) ordenó las muestras en forma similar que el dendrograma de similitud.

\section{Discusión}

El fitoplancton de Bahía Independencia durante el período estudiado, como es típico en un área de surgencia presentó una alta producción, dominancia de diatomeas pequeñas de rápido crecimiento que se unen en cadena y tipifican la primera fase de la sucesión, con índices de diversidad bajos que corresponden a poblaciones en activo crecimiento y con pocas especies dominantes (Margalef 1958, 1968 y 1978). Estas características son concordantes con lo reportado por Blasco (1971), Rojas de Mendiola y Estrada (1976), Ochoa y Gómez (1981 y 1988), Rojas de Mendiola (1981), Delgado y Villanueva (1998) y Chang et al. (1999) en estudios a nivel mesoescala en las aguas costeras de la Corriente Peruana.

Sánchez et al. (1988) en el invierno de 1986 y Antonietti (1989) en abril de 1987 encontraron para el área de Bahía Independencia la misma composición especiológica con predominancia de $T$. nitzschioides y frecuencia de $S$. costatum, A. glacialis, D. pumila, $G$. marina y Pleurosigma. Estos resultados permiten establecer una constancia en la composición específica del fitoplancton de esta área.

La sustitución cronológica en la dominancia de las especies $S$. costatum y $T$. nitzschioides muestra un ligero avance en la sucesión fitoplanctónica, sin alcanzar el climax. $S$. costatum es una especie típica de la etapa inicial y $T$. nitzschioides es típica de una etapa algo más avanzada de la sucesión (Blasco, 1971).La alta variabilidad de las poblaciones fitoplanctónicas en cortos períodos de tiempo encontrada en este estudio coincide con lo reportado para otras áreas costeras someras,

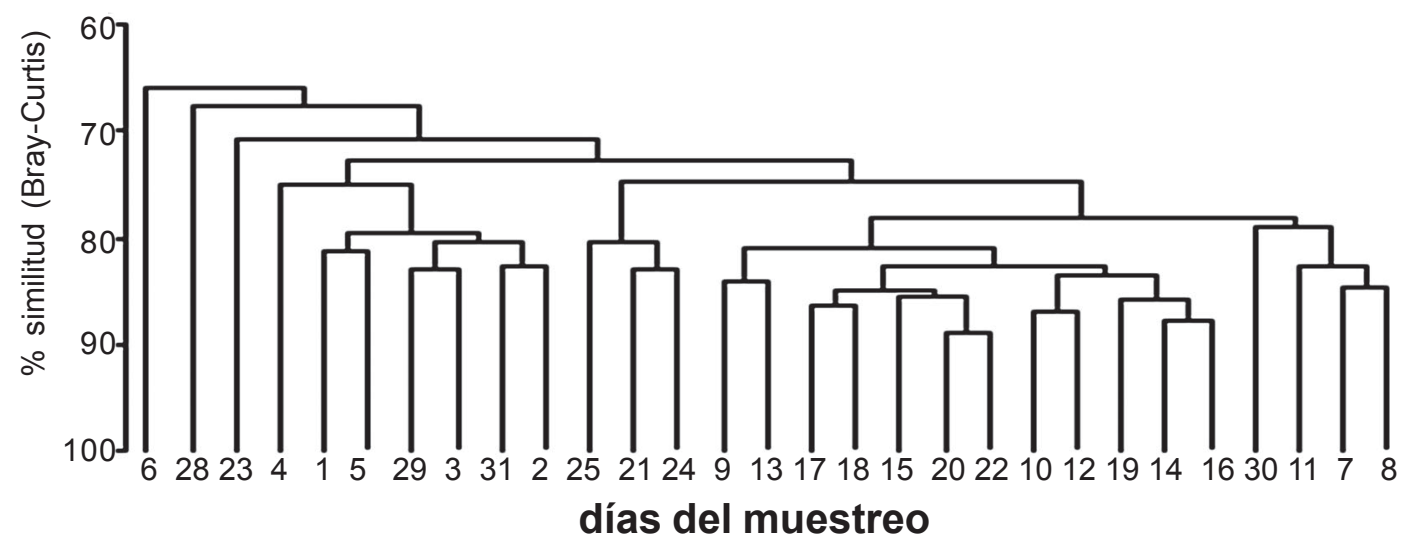

Figura 9. Dendrograma de similitud entre promedios diarios de densidad del total de fitoplancton en Bahía Independencia. Se utilizó el índice de Bray-Curtis. Los números indican los dias de muestreo en los meses de marzo y abril 1988, (28 de marzo - 25 de abril). 


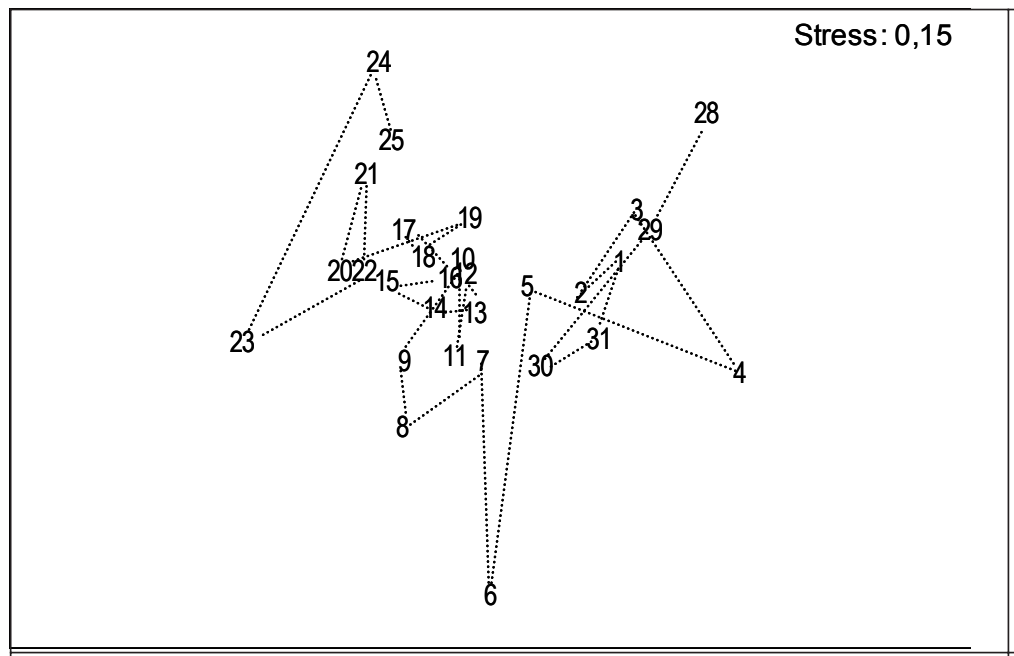

Figura 10. Ordenación NMDS en base a los valores de promedios diarios de densidad del total de fitoplancton. Los números indican los dias de muestreo en los meses de marzo y abril 1988, (28 de marzo -25 de abril).

Harrison et al. (1981) para Chimbote, Alvial y Avaria (1982) para las aguas costeras de Chile, Abboud-Abi Saab (1992) para aguas costeras libanesas.

Aún cuando la temperatura no varió mucho durante la investigación, los incrementos de fitoplancton están asociados con los pequeños incrementos de temperatura.

\section{Conclusiones}

Las aguas de bahía Independencia son altamente productivas con un fitoplancton típico de áreas de afloramiento costero, con predominancia de diatomeas pequeñas, baja diversidad y con fuertes variaciones de densidad en cortos períodos de tiempo.

La estructura comunitaria corresponde a un estado inicial de la sucesión.

\section{Literatura citada}

Abboud-Abi Saab, M. 1992. Day-to-day variation in phytoplankton assemblages during spring blooming in a fixed station along the Lebanese coastline. J. Plankton Research, 14(8): 10991115.

Alveal, A. y S. Avaria. 1982. Proliferación de primavera del fitoplancton en la Bahía de Valparaíso. II. Dinámica de las comunidades. Rev. Biol. Mar., Valparaíso, 18(1): 1-56.

Antonietti, E. 1989. El fitoplancton en dos bahías del litoral peruano en abril 1987. Mem. Simp. Internacional de los Recursos Vivos y las Pesquerías en el Pacífico Sudeste. Viña del Mar. Com. Permanente del Pacífico Sur. Rev. Pacífico Sur. Número Especial.
Barreda, M. 1958. El plancton de la Bahía de Pisco. Bol. Comité de Protección a la Naturaleza, 16: 61-106.

Blasco, D. 1971. Composición y distribución del fitoplancton en la región de afloramiento de las costas peruanas. Inv. Pesq., 35: 61-112.

Chang, F.; E. Delgado y C. Fernández.1999. Composición, Abundancia y distribución del fitoplancton frente a la costa del Perú durante el verano 1999. Inf. Inst. Mar Perú, 147: 99-108.

Delgado, E. y P. Villanueva. 1998. La comunidad fitoplanctónica en el litoral peruano durante marzo-mayo 1998. Crucero BIC Humboldt 9803-05 de Tumbes a Tacna. Inf. Inst. Mar Perú, 135: 114-120.

Harrison, W. G., T. Platt, R. Calienes and N. Ochoa. 1981. Photosynthetic parameters and primary production of phytoplankton population off the Northern Coast of Perú. In F. A. Richards (ed.): Coastal Upwelling. American Geophysical Union, Washington D. C., 303-311.

Margalef, R. 1958. Temporal succesión and spatial heterogeneity in phytoplankton. In: BuzzatiTraverso (ed.), Perspectives in Marine Biology, Univ. Calif. Press, Berkeley, Los Angeles, Calif. 323-349.

Margalef, R. 1968. Perspectives in ecological Theory, The University of Chicago press. Chicago, $111 \mathrm{pp}$.

Margalef, R. 1978. Phytoplankton communities in Upwelling areas. The example of NW Africa. Oecol, Aquat., 3: 97-132.

Ochoa, N. y O. Gómez. 1981. Variaciones del fitoplancton en el área de Chimbote durante 1977. In: A. Landa (ed): Investigación Cooperativa de la Anchoveta y su Ecosistema (ICANE) entre Perú y Canadá. Bol. Inst. Mar Perú, Vol. Extraordinario, 119-129.

Ochoa, N. y O. Gómez. 1988. Variación espacio temporal del fitoplancton frente al Callao, Perú, en 1986. En: H. Salzwedel y A. Landa (eds): Re- 
cursos y dinámica del ecosistema de afloramiento peruano. Bol. Inst. Mar Perú. Vol. Extraordinario, 51-57.

Rojas de Mendiola, B. 1981. Seasonal phytoplankton distribution along the Peruvian coast. I: F. A. Richards (ed.), Coastal Upwelling. American Geophysical Union, Washington D. C., 348356.

Rojas de Mendiola, B. y M. Estrada. 1976. E1 fitoplancton en el área de Pimentel. Verano de 1972. Inv. Pesq., 40: 463-490.

Sánchez, S.; J. Tarazona, R. Flores, M. Maldonado y G. Carbajal. 1988. Características del fitoplancton de invierno en Bahía Independencia, Perú. En: H. Salzwedel y A. Landa (eds.): Recursos y Dinámica del ecosistema de afloramiento peruano. Bol. Inst. Mar Perú. Vol. Extraordinario, 57-66.

Shannon, C. y Weaner. 1963. The mathematical theory of communication. University of Illinois Press, Urbana. 125 pp.
Sneath, P. H. A. y R. R. Sokal. 1973. Numerical taxonomy. W. H. Freeman, San Francisco. 571 pp.

Tarazona, J.; H. Santander, S. Sánchez, S. Carrasco y S. Guzmán. 1989. Características del plancton. En: J. Tarazona, C. Paredes y L. Romero (eds.): Mecanismos y procesos que controlan la colonización y recuperación postcatastrófica de recursos bentónicos de importancia económica en dos áreas de diferente productividad del sistema de afloramiento peruano. Univ. Nac. Mayor de San Marcos. Fac. Ciencias Biológicas, 68-169.

UNESCO. 1978. Phytoplankton Manual. Monogr. Oceanogr. Methodology, 6, 337 pp.

Utermöhl, H. 1958. Zur Vervollkommung der quantitativen Phytoplankton Methodik. Mitt. Int. Ver. Theor. angew. Limnol., 9: 1-38. 